\title{
The epidemiology of polypharmacy
}

\author{
Author: Rupert A Payne ${ }^{A}$
}

Polypharmacy describes, in simplistic terms, the use of multiple medications in an individual. It has become a normal aspect of modern medicine, driven by an ageing, multimorbid population, the increasing availability of preventative medications and an increasing use of single-disease guidelines and adherence to evidence-based practice. However, polypharmacy is also associated with a range of adverse outcomes, and is considered an important and increasing challenge for clinical practice. Here, we consider the definitions of polypharmacy, the extent and nature of medication use in different settings, and the type of problems encountered as a consequence of polypharmacy.

\section{Introduction}

Polypharmacy is considered an important and growing challenge for modern clinical practice, attracting interest from clinicians, guideline developers and policymakers alike. Here, we consider the magnitude of the problem in the general adult population from an epidemiological perspective, drawing on evidence from a narrative review of the literature.

\section{Definition of polypharmacy}

The word polypharmacy is derived from the ancient Greek 'polús' meaning 'many', and 'pharmakeía' meaning 'the use of drugs'. This broad meaning from a purely linguistic perspective is reflected in the fact that there is no consensus on a clinical definition of polypharmacy. For example, should the term refer to only simultaneous opposed to consecutive medications, include short-term as well as long-term therapy, be restricted to prescription-only medications, or include non-pharmacological products? These points make it difficult to accurately quantify the extent of the issue or enable comparisons between health systems or care settings.

One of the most commonly employed definitions of polypharmacy in practice is the number of medications exceeding a simple numeric threshold (although how medications are counted is subject to the issues above). Although having the advantage of simplicity, polypharmacy is a continuum and dichotomising in this way is of limited value

Author: ${ }^{A}$ consultant senior lecturer in primary healthcare, Centre for Academic Primary Care, University of Bristol, Bristol, UK in assessing drug-related problems. ${ }^{1}$ Furthermore, increased medication use in current practice means that previously relevant cut-offs such as 'five or more drugs' may no longer be appropriate.

An even more important limitation to the use of simple thresholds is a failure to capture the appropriateness or otherwise of treatment. A King's Fund report on the subject advocated classification of polypharmacy as either appropriate or problematic. ${ }^{2}$ The former was defined as 'prescribing for an individual for complex conditions or for multiple conditions in circumstances where medicines use has been optimised and the medicines are prescribed according to best evidence', with the intent of improving quality of life and longevity while minimising harm. Problematic polypharmacy was defined as 'where multiple medications are prescribed inappropriately, or where the intended benefit of the medication is not realised', potentially through non-evidence-based therapy, unfavourable risk-benefit balance, hazardous interactions, unacceptable treatment burden or poor adherence, or an undesirable prescribing cascade.

\section{Key points}

Polypharmacy is a common issue encountered in both primary and secondary care, and should be differentiated into appropriate or problematic

There is no accepted means of measuring either magnitude or appropriateness of polypharmacy. Nevertheless, a range of clinical indicators exist for the identification of potentially inappropriate prescribing, which are relevant (but not specific) to problematic polypharmacy

Increasing age, multimorbidity and widespread use of evidence-based clinical guidelines are strongly associated with increased polypharmacy

Polypharmacy is associated with a range of adverse clinical outcomes, but the use of multiple medications is not always harmful and the clinical context of treatment should be considered

KEYWORDS: Multimorbidity, pharmacoepidemiology, polypharmacy, potentially inappropriate prescribing 
However, this qualitative definition is not straightforward to implement in clinical informatics systems and not readily applicable to epidemiological studies. A 2014 systematic review identified 46 different tools for assessing inappropriate prescribing, the majority (36) focusing on the older population. ${ }^{3}$ Tools were categorised as explicit, criterion-based tools (eg Beers' criteria, ${ }^{4}$ STOPP (Screening Tool of Older People's potentially inappropriate Prescriptions)/START (Screening Tool to Alert doctors to Right Treatment) criteria ${ }^{5}$ ), implicit, judgement-based tools (eg Medication Appropriateness Index ${ }^{6}$ ), or a combination (Box 1). Explicit criteria are lists of potentially inappropriate prescribing specific to particular drugs, generally identified through expert consensus. These criteria have the advantage of being readily implemented into

\section{Box 1. Indicators of potentially inappropriate prescribing}

Selected examples of explicit indicators from the STOPP criteria $^{5}$

Beta-blocker in combination with verapamil or diltiazem

Aspirin with a past history of peptic ulcer disease without concomitant PPI

Initiation of tricyclic antidepressants as first-line antidepressant treatment

Direct thrombin inhibitors (eg dabigatran) if eGFR $<30 \mathrm{~mL} /$ $\min / 1.73 \mathrm{~m}^{2}$

Systemic corticosteroids instead of inhaled corticosteroids for maintenance therapy in moderate-severe COPD

NSAID with established hypertension or heart failure

Selective alpha-1 selective alpha blockers in those with symptomatic orthostatic hypotension

Oestrogens with a history of breast cancer or venous thromboembolism

Use of regular (as distinct from PRN) opioids without concomitant laxative

Full list of implicit indicators from the Medication Appropriateness Index ${ }^{6}$

Is there an indication for the drug?

Is the medication effective for the condition?

Is the dosage correct?

Are the directions correct?

Are the directions practical?

Are there clinically significant drug-drug interactions?

Are there clinically significant drug-disease/condition interactions?

Is there unnecessary duplication with other drug(s)?

Is the duration of therapy acceptable?

Is this drug the least expensive alternative compared to others of equal utility?

COPD = chronic obstructive pulmonary disease; eGFR = estimated glomerular filtration rate; NSAID = Nonsteroidal anti-inflammatory drug; $\mathrm{PPI}=$ proton pump inhibitor; PRN = pro re nata (when required); STOPP = Screening Tool of Older People's potentially inappropriate Prescriptions automated systems, but are restricted to finite numbers of drug- or disease-specific problems. In contrast, implicit criteria can be applied to any medication, but generally require human evaluation and are relatively subjective. None of these tools are specifically designed for measuring problematic polypharmacy although implicit tools are arguably better suited to this by capturing the entirety of the patient's medication regimen; a handful of such indicators have been devised for optimising polypharmacy. $^{3}$

\section{Extent of polypharmacy in the UK and beyond}

In a study by Guthrie and colleagues, ${ }^{7}$ the numbers of Scottish adults dispensed 5-9, 10-14, or 15 or more medications in primary care over a 3 -month period in 2010 were $16.3 \%, 4.7 \%$ and $1.1 \%$, respectively. These figures had increased from $9.7 \%$, $1.5 \%$ and $0.2 \%$, respectively, in 1995 . The most frequently prescribed medications are shown in Fig 1. Just under a quarter of adults were dispensed medications from three or more chapters of the British National Formulary. ${ }^{7}$ Importantly, these data do not distinguish appropriate from problematic polypharmacy, although rates of inappropriate prescribing have been reported; a UK study found that the prevalence of potentially inappropriate prescribing (a composite of 13 indicators) in all patients at risk of exposure was around $5 \%{ }^{8}$ and an Irish study using 30 STOPP criteria reported a prevalence of over a third in older patients. ${ }^{9}$ Differences in the way data are captured and polypharmacy is defined makes international comparison challenging but the issue is nevertheless a global one. $11 \%$ of the Swedish primary care population ${ }^{10}$ and $6 \%$ of a rural Chinese population were in receipt of five or more medications; ${ }^{11}$ two-thirds of over-60s in a Brazilian primary care study received four or more medications. ${ }^{12}$

Age and multimorbidity are important factors driving polypharmacy. The UK population is predicted to grow by $15 \%$ over the next 25 years, with 1 in 12 people being over the age of 80 years by $2039,{ }^{13}$ and approximately a quarter of the UK adult population have two or more long-term health conditions. ${ }^{14}$ Fig 2 summarises evidence from another Scottish study showing polypharmacy to have a strong positive association with both number of clinical conditions and increasing age. ${ }^{15}$ Patients in receipt of higher numbers of medications are also more likely to be female, more socio-economically deprived and live in care homes. ${ }^{7,15}$ There is considerable variation in types of conditions for which multiple medication use is observed, with the highest level of prescribing observed in cardiovascular disease, clinically unrelated problems and other conditions managed using complex medication regimens. ${ }^{15}$

Clinical guidelines play an increasingly major role in clinicians' therapeutic decision making around long-term conditions. However, most guidelines are designed with single diseases in mind, rather than the holistic care of patients. It is difficult to quantify the effect of guidelines on the growth of polypharmacy. In the UK, the National Institute for Health and Care Excellence has published over 170 clinical guidelines to date; this has increased from three new guidelines in 2004 to 21 in 2015. There is evidence that such guidelines impact directly on clinicians' prescribing behaviours ${ }^{16}$ and a study examining the potential impact of guideline adherence in a hypothetical clinical situation found this would lead to considerable treatment burden. ${ }^{17}$ 


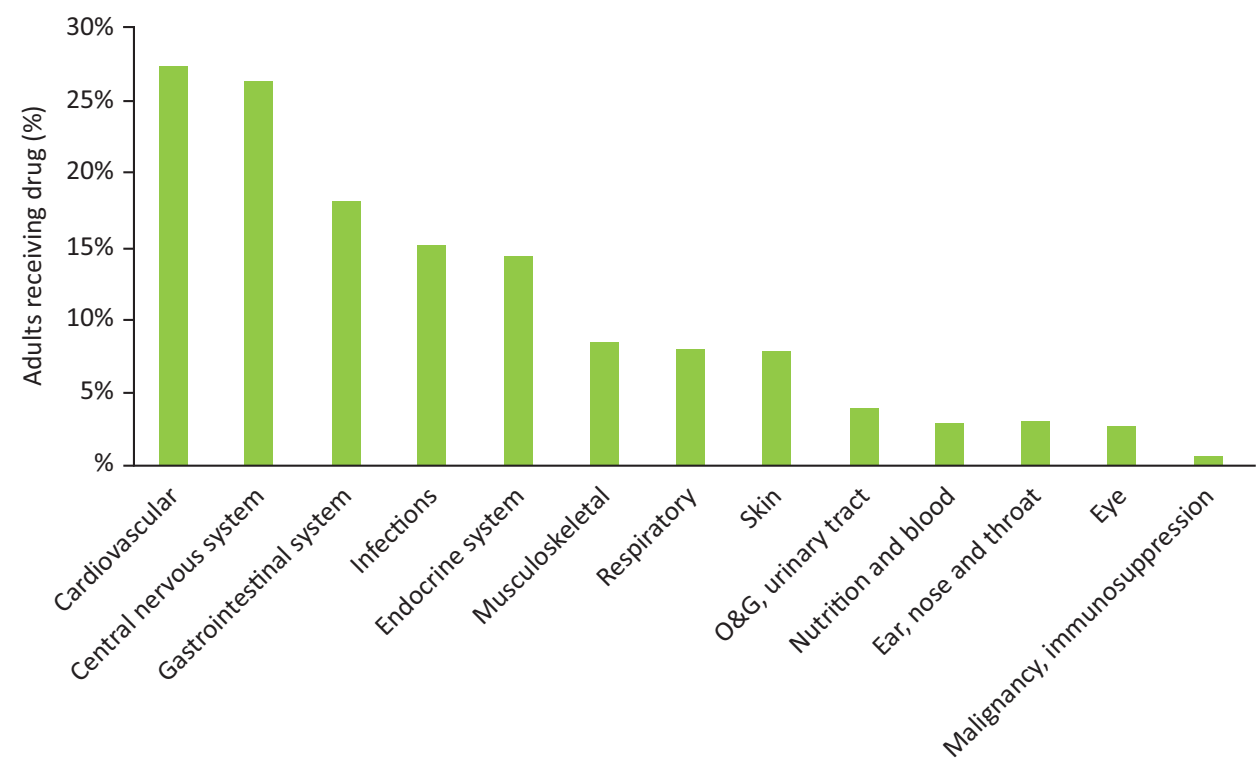

Fig 1. Percentages of adults prescribed medications in Scottish primary care in 2010 according to chapter in the British National Formulary. $O \& G=$ obstetrics and gynaecology. Data from Guthrie et al. ${ }^{7}$

Admission to hospital is an important driving factor behind polypharmacy, although UK data is lacking. A cross-European study by Gallagher and colleagues reported the median number of medications in older inpatients to be 6 (interquartile range $4-9) .{ }^{18}$ A number of studies have described the impact of hospitalisation upon prescribing rates; an Australian study reported an increase following acute admission to an elderly care unit of 6.6 to 7.7 long-term medications, most marked in younger patients (65-75 years). ${ }^{19}$ Importantly, considerable changes are often made to medication regimens in the weeks and months after discharge, with one study finding this affected $86 \%$ of patients. $^{20}$

\section{Consequences of polypharmacy}

Medication safety issues are common and there is strong evidence that these are associated with polypharmacy. Commissioned by the General Medical Council, the PRACtICe (PRevalence And Causes of prescrIbing errors in general practiCe) study of UK general practice prescribing errors found the odds of a medication error increased by $16 \%$ for each additional medication prescribed, although medication appropriateness was not considered. ${ }^{21}$ Prescribing or monitoring errors were observed in one in eight patients, affecting $5 \%$ of all prescription items. The total number
A

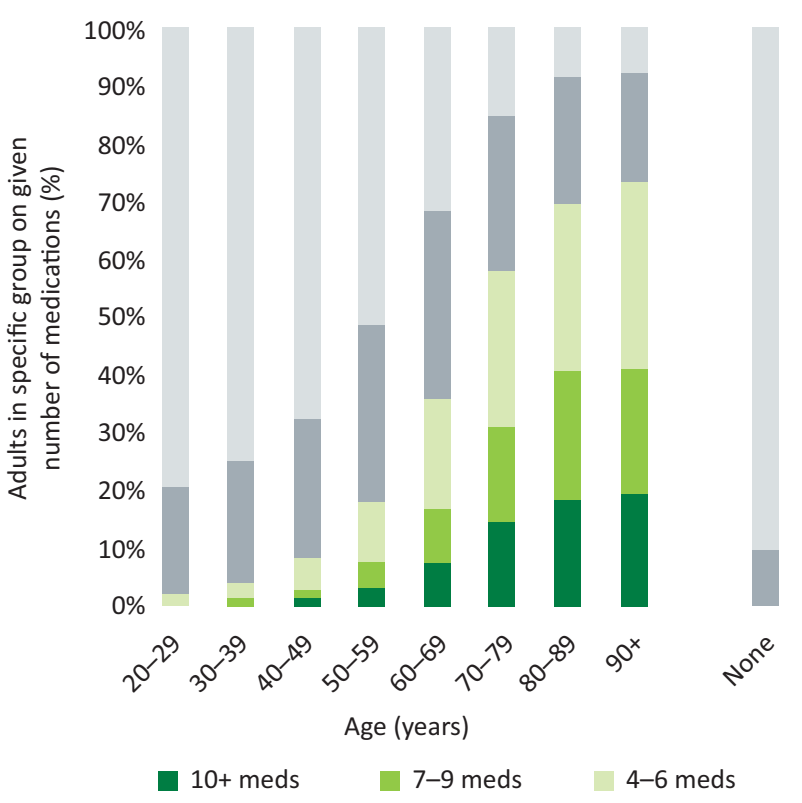

B

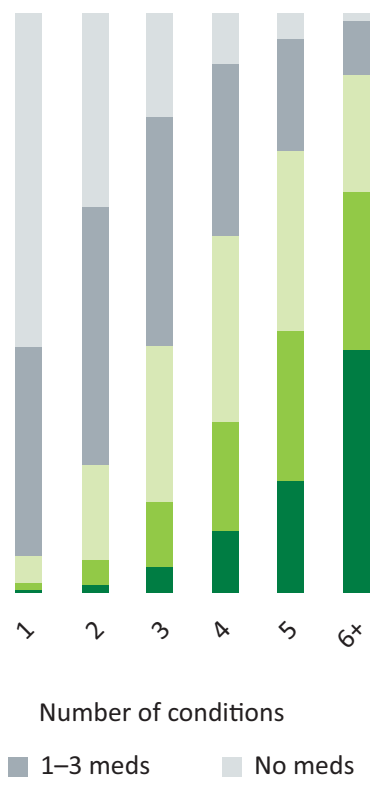

Fig 2. Percentage of Scottish adults on given number of medications by age (A) and number of conditions (B). Adapted with permission from Payne et al. ${ }^{15}$ 
Both sets of guidance provide basic principles, supported by summaries of medication effectiveness, disease-specific recommendations and advice on specific clinically-challenging areas.

Welsh guidance ${ }^{31}$ recommends the use of the NO TEARS model for medication review ${ }^{32}$ and a focus on high-risk medication, with specific advice on medication adherence, deprescribing and assessment of whether patients can manage their medications. Particular consideration is given to groups with large numbers of medications, indications of shortened life expectancy, and frail and elderly patients.

Scottish guidance ${ }^{30}$ is based around the following seven-step approach to medication review:

$\begin{array}{ll}\text { Domain } & \text { Steps } \\ \text { Aims } & 1 \text { Identify objectives of drug therapy } \\ \text { Need } & 2 \text { Identify essential drug therapy } \\ & 3 \text { Does the patient take unnecessary drug therapy } \\ \text { Effectiveness } & 4 \text { Are therapeutic objectives being achieved? } \\ \text { Safety } & 5 \text { Does the patient have ADR or is at risk of ADRs? } \\ \text { Cost-effectiveness } & 6 \text { Is drug therapy cost-effective? } \\ \text { Concordance } & 7 \text { Is the patient willing and able to take drug therapy as intended? }\end{array}$

$\mathrm{ADR}=$ adverse drug reaction

of medications is also strongly associated with high-risk prescribing; there is a three-fold higher risk for patients with 10 long-term medications compared with one or two medications. ${ }^{22}$ Potentially serious drug-drug interactions occur in $13 \%$ of patients; $1.2 \%$ of patients have four or more such interactions. Over two thirds of patients receiving 10 or more medications have a potentially serious interaction. ${ }^{7}$ Moriarty and colleagues found the receipt of five or more regular medications was associated with a near 7 -fold increase in the odds of potentially inappropriate prescribing although, interestingly, the risk presented by polypharmacy has decreased significantly over time. ${ }^{9}$

Polypharmacy, as quantified by simple medication count, is also associated with increased health service utilisation. Adverse drug reactions have been shown to account for $6.5 \%$ of hospital admissions, ${ }^{23}$ with polypharmacy the principal driving factor. ${ }^{24}$ Polypharmacy is also used in a number of predictive models of unplanned hospitalisation in general. ${ }^{25}$ However, it is necessary to recognise that the latter relationship is driven strongly by the close correlation between polypharmacy and morbidity. An observational study by Payne and colleagues found the strength of association between polypharmacy and admission to be considerably attenuated in the most multimorbid individuals, consistent with therapeutic benefit. ${ }^{26}$ This once again highlights the importance of differentiating appropriate from problematic polypharmacy.

Of course, although pharmacotherapy has obvious clinical benefits in many situations, it is important to consider adverse consequences not simply related to safety. In particular, medication adherence has been consistently shown to be lower in those patients in receipt of multiple medications, ${ }^{27}$ with the potential consequent risk of reducing the efficacy of treatments. Furthermore, there is evidence that perceived over-medication is associated with poorer reported health-related quality of life, ${ }^{28}$ again potentially undermining some of the therapeutic benefit.

Although outside the scope of this article, given the potential adverse consequences of problematic polypharmacy, it is worth briefly considering relevant medication optimisation interventions. A recent Cochrane review identified 12 interventions designed to improve appropriate use of polypharmacy in older patients. ${ }^{29}$ The majority of these were multifaceted complex interventions delivered by pharmacists, and provided at various points in the care pathway. Intervention components included medication review, patient education, health professional training, medication adherence aids and multidisciplinary case conferences. Studies were of limited quality and focused on reducing medication count as opposed to prescribing appropriateness. Reductions in inappropriate prescribing were observed although there was a lack of evidence of benefit in terms of clinical outcomes. National guidance has been issued on the management of polypharmacy in both Scotland ${ }^{30}$ and Wales ${ }^{31}$, and key aspects of these are summarised in Box 2.

\section{Conclusion}

Polypharmacy is a growing and global issue, affecting primary and secondary care. It is predominantly driven by an ageing, multimorbid population coupled with the increasing use of evidence-based clinical guidelines. Clinicians need to be aware of the range of problems it can lead to and ensure the benefits of using multiple medications are balanced appropriately against the potential harms.

\section{Conflicts of interest}

RAP is a member of the NICE Guideline Development Group for multimorbidity and has received honoraria for speaking on the topic of polypharmacy.

\section{References}

1 Viktil KK, Blix HS, Moger TA, Reikvam A. Polypharmacy as commonly defined is an indicator of limited value in the assessment of drug-related problems. Br J Clin Pharmacol 2007;63:187-95.

2 Duerden M, Avery AJ, Payne RA. Polypharmacy and medicines optimisation: making it safe and sound. London: The King's Fund, 2013. 
3 Kaufmann CP, Tremp R, Hersberger KE, Lampert ML. Inappropriate prescribing: a systematic overview of published assessment tools. Eur J Clin Pharmacol 2014;70:1-11.

4 American Geriatrics Society 2015 Beers Criteria Update Expert Panel. American Geriatrics Society 2015 Updated Beers Criteria for Potentially Inappropriate Medication Use in Older Adults. J Am Geriatr Soc 2015;63:2227-46.

5 O'Mahony D, O’Sullivan D, Byrne S et al. STOPP/START criteria for potentially inappropriate prescribing in older people: version 2 . Age Ageing 2015;44:213-8.

6 Hanlon JT, Schmader KE, Samsa GP et al. A method for assessing drug therapy appropriateness. J Clin Epidemiol 1992;45:1045-51.

7 Guthrie B, Makubate B, Hernandez-Santiago V, Dreischulte T. The rising tide of polypharmacy and drug-drug interactions: population database analysis 1995-2010. BMC Med 2015;13:74.

8 Stocks SJ, Kontopantelis E, Akbarov A et al. Examining variations in prescribing safety in UK general practice: cross sectional study using the Clinical Practice Research Datalink. BMJ 2015;351:h5501.

9 Moriarty F, Hardy C, Bennett K, Smith SM, Fahey T. Trends and interaction of polypharmacy and potentially inappropriate prescribing in primary care over 15 years in Ireland: a repeated crosssectional study. BMJ Open 2015;5:e008656.

10 Hovstadius B, Hovstadius K, Astrand B, Petersson G. Increasing polypharmacy - an individual-based study of the Swedish population 2005-2008. BMC Clin Pharmacol 2010;10:16.

11 Dong L, Yan H, Wang D. Polypharmacy and its correlates in village health clinics across 10 provinces of Western China. J Epidemiol Community Health 2010;64:549-53.

12 Oliveira MG, Amorim WW, de Jesus SR, Rodrigues VA, Passos LC. Factors associated with potentially inappropriate medication use by the elderly in the Brazilian primary care setting. Int J Clin Pharm 2012;34:626-32.

13 Office for National Statistics. National Population Projections: 2014 based Statistical Bulletin. London: Office for National Statistics, 2015.

14 Barnett K, Mercer SW, Norbury M et al. Epidemiology of multimorbidity and implications for health care, research, and medical education: a cross-sectional study. Lancet 2012;380:37-43.

15 Payne RA, Avery AJ, Duerden M et al. Prevalence of polypharmacy in a Scottish primary care population. Eur J Clin Pharmacol 2014;70:575-81.

16 Thornhill MH, Dayer MJ, Forde JM et al. Impact of the NICE guideline recommending cessation of antibiotic prophylaxis for prevention of infective endocarditis: before and after study. BMJ 2011;342:d2392.

17 Hughes LD, McMurdo ME, Guthrie B. Guidelines for people not for diseases: the challenges of applying UK clinical guidelines to people with multimorbidity. Age Ageing 2013;42:62-9.

18 Gallagher P, Lang PO, Cherubini A et al. Prevalence of potentially inappropriate prescribing in an acutely ill population of older patients admitted to six European hospitals. Eur J Clin Pharmacol 2011;67:1175-88
19 Betteridge TM, Frampton CM, Jardine DL. Polypharmacy-we make it worse! A cross-sectional study from an acute admissions unit. Intern Med J 2012;42:208-11.

20 Viktil KK, Blix HS, Eek AK et al. How are drug regimen changes during hospitalisation handled after discharge: a cohort study. BMJ Open 2012;2:e001461.

21 Avery AJ, Barber N, Ghaleb $\mathrm{M}$ et al. Investigating the prevalence and causes of prescribing errors in general practice: The PRACtICe Study. London: GMC, 2012.

22 Guthrie B, McCowan C, Davey P et al. High risk prescribing in primary care patients particularly vulnerable to adverse drug events: cross sectional population database analysis in Scottish general practice. BMJ 2011;342:d3514.

23 Pirmohamed M, James S, Meakin S et al. Adverse drug reactions as cause of admission to hospital: prospective analysis of 18820 patients. BMJ 2004;329:15-9.

24 Davies EC, Green CF, Taylor S et al. Adverse drug reactions in hospital in-patients: a prospective analysis of 3695 patient-episodes. PLoS One 2009;4:e4439.

25 The King's Fund. Combined predictive model: Final report. London: Health Dialog UK, The King's Fund, New York University, 2006.

26 Payne RA, Abel GA, Avery AJ, Mercer SW, Roland MO. Is polypharmacy always hazardous? A retrospective cohort analysis using linked electronic health records from primary and secondary care. Br J Clin Pharmacol 2014;77:1073-82.

27 Gellad WF, Grenard JL, Marcum ZA. A systematic review of barriers to medication adherence in the elderly: looking beyond cost and regimen complexity. Am J Geriatr Pharmacother 2011;9:11-23.

28 Fincke BG, Miller DR, Spiro A 3rd. The interaction of patient perception of overmedication with drug compliance and side effects. J Gen Intern Med 1998;13:182-5.

29 Cooper JA, Cadogan CA, Patterson SM et al. Interventions to improve the appropriate use of polypharmacy in older people: a Cochrane systematic review. BMJ Open 2015;5:e009235.

30 Scottish Government Model of Care Polypharmacy Working Group. Polypharmacy Guidance, 2nd edn. Edinburgh: Scottish Government, 2015.

31 All Wales Medicines Strategy Group. Polypharmacy: Guidance for prescribing. Llandough: All Wales Medicines Strategy Group, 2014.

32 Lewis T. Using the NO TEARS tool for medication review. BMJ 2004;329:434.

Address for correspondence: Dr R A Payne, Centre for Academic Primary Care, School of Social and Community Medicine, University of Bristol, Canynge Hall, 39 Whatley Road, Bristol BS8 2PS, UK.

Email: r.payne@bristol.ac.uk 